\title{
Carbachol-Mediated Endocytosis of NHE3 Involves a Clathrin-Independent Mechanism Requiring Lipid Rafts and Cdc42
}

\author{
Nicholas C. Zachos Bharath Alamelumangpuram Luke J. Lee Peng Wang \\ Olga Kovbasnjuk
}

Department of Medicine/Gastroenterology and Hepatology, Johns Hopkins University School of Medicine, Baltimore, MD, USA

\section{Key Words}

Protein trafficking • Intestinal epithelial cells $•$ Intracellular calcium

\begin{abstract}
Background: In intestinal epithelial cells, acute regulation of the brush border $\mathrm{Na}^{+} / \mathrm{H}^{+}$ exchanger, NHE3, usually occurs by changes in endocytosis and/or exocytosis. Constitutive NHE3 endocytosis involves clathrin. Carbachol $(\mathrm{CCH})$, which elevates intracellular $\mathrm{Ca}^{2+}$ $\left(\left[\mathrm{Ca}^{2+}\right]\right.$.), decreases NHE3 activity and stimulates endocytosis; however, the mechanism involved in calcium-mediated endocytosis of NHE3 is unclear. A pool of NHE3 resides in lipid rafts, which contributes to basal, but not CAMP-mediated, NHE3 trafficking, suggesting that an alternative mechanism exists for NHE3 endocytosis. Cdc42 was demonstrated to play an integral role in some cases of cholesterol-sensitive, clathrin-independent endocytosis. Therefore, the current study was designed to test the hypotheses that (1) clathrin-mediated endocytosis (CME) is involved in constitutive, but not $\mathrm{CCH}$-mediated, endocytosis of NHE3, and (2) $\mathrm{CCH}$-mediated endocytosis of NHE3 occurs through a lipid raft, activated Cdc42-dependent pathway that does not involve clathrin. Methods: The role of Cdc42 and lipid rafts on NHE3 activity and endocytosis were investigated in polarized Caco-2/BBe cells using pharmacological and shRNA knockdown approaches. Results: Basal NHE3 activity was increased in the presence of CME blockers (chlorpromazine; $\mathrm{K}^{+}$depletion) supporting previous reports that constitutive $\mathrm{NHE3}$ endocytosis is clathrin dependent. In contrast, $\mathrm{CCH}$-inhibition of NHE3 activity was abolished in Caco-2/BBe cells treated with $M \beta C D$ (to disrupt lipid rafts) as well as in Cdc42 knockdown cells but was unaffected by CME blockers. Conclusion: $\mathrm{CCH}$-mediated inhibition of NHE3 activity is not dependent on clathrin and involves lipid rafts and requires Cdc42.
\end{abstract}




\section{Introduction}

The BB Na+$/ \mathrm{H}^{+}$Exchanger, NHE3, accounts for most of the electroneutral $\mathrm{Na}^{+}$absorption that occurs during digestion. NHE3 is initially inhibited in digestion and later in digestion is stimulated [1]. In most diarrheal diseases, there is inhibition of neutral $\mathrm{NaCl}$ absorption in the $\mathrm{Na}^{+}$absorptive cell primarily due to NHE3 inhibition. In the intact ileum, carbachol (CCH) elevated intracellular calcium $\left(\left[\mathrm{Ca}^{2+}\right]_{\mathrm{i}}\right)$ via basolateral membrane (BLM) muscarinic receptors (M3) which inhibited NHE3 activity by $40 \%$ and similarly decreased surface expression of NHE3 [2-5]. NHE3 is often regulated by changes in its plasma membrane versus intracellular location as a result of changes in the rates of endocytosis and/or exocytosis [6-14]. Basal NHE3 endocytosis occurs by a clathrin-dependent process which partially involves lipid rafts $[15,16]$. Previous studies demonstrated that constitutive and cAMP regulated NHE3 endocytosis occurs through a clathrin-dependent process $[9,17]$. Carbachol elevation of $\left[\mathrm{Ca}^{2+}\right]_{\mathrm{i}}$ decreases NHE3 activity and stimulates endocytosis as early as one minute; however, studies examining $\left[\mathrm{Ca}^{2+}\right]_{\mathrm{i}}$-mediated endocytosis of NHE3 in polarized intestinal epithelial cells are incomplete [14]. A pool of BB NHE3 resides in lipid rafts which contributes to basal, but not cAMP-mediated, NHE3 trafficking, suggesting an alternative mechanism exists for NHE3 endocytosis $[15,16]$. Recently, the Rho GTPase, Cdc42, has been demonstrated to play an integral role in cholesterol-sensitive, clathrin-independent endocytosis of integral membrane proteins including the folate receptor as well as the Helicobacter pylori VacA cytotoxin [18-20]. Cdc42 is necessary for a clathrin-independent endocytic process termed the CLathrin Independent Carrier (CLIC)/GPI-anchored protein-enriched Early Endocytic Compartment (GEEC) pathway [21, 22]. Previous studies designed to understand the role of elevated $\left[\mathrm{Ca}^{2+}\right]_{i}$ on NHE3 trafficking have used non-physiologic agents including calcium ionophores and thapsigargin in fibroblast or non-polarized epithelial cells [17, 23-25]. This study tested the hypotheses that: (1) inhibition of clathrin-mediated endocytosis (CME), prevents basal but not CCH-mediated, endocytosis of NHE3, and (2) CCH-mediated endocytosis of NHE3 occurs through a lipid raft and activated Cdc42-dependent pathway that does not involve clathrin.

\section{Materials and Methods}

\section{Reagents}

Carbachol (CCH), chlorpromazine (CPZ), methyl- $\beta$-cyclodextrin (M $\beta C D$ ), and BAPTA-AM were from Sigma. Pirl-1 (8-cyclohexil-5,6-dihidro-4H-pyrazino[3,2,1-jk]carbazole), which inhibits guanine nucleotide exchange on Cdc42, was from Chembridge (San Diego, CA) [26]. AlexaFluor 488 conjugated wheat germ agglutinin (WGA) was from Invitrogen.

\section{Antibodies}

Affinity-purified rabbit polyclonal antibodies to Cdc42 and clathrin heavy chain were from Cell Signaling. Polyclonal caveolin-1 antibody was from Santa Cruz. Unconjugated monoclonal anti-HA antibody was from Covance. AlexaFluor 594 conjugated anti-HA antibody was from Invitrogen.

\section{Cell Line}

Caco-2/BBe cells express all four members of the NHERF gene family and small amounts of NHE3 [27]. Triple HA-tagged rabbit NHE3 was transiently expressed by adenovirus into Caco-2/BBe cells for transport and biochemical analysis. Caco-2/BBe cells were grown on Anapore filters (Nunc) until post-confluent for 12 days in Dulbecco's modified Eagle's medium supplemented with 25mM $\mathrm{NaHCO}_{3}$, 10mM HEPES, $0.1 \mathrm{mM}$ nonessential amino acids, 50 units $/ \mathrm{ml}$ penicillin, $50 \mu \mathrm{g} / \mathrm{ml}$ streptomycin, and $10 \%$ fetal bovine serum in a $5 \% \mathrm{CO}_{2}, 95 \%$ air incubator at $37^{\circ} \mathrm{C}$. Cells were serum starved overnight and then treated with $6 \mathrm{mM}$ EGTA for $2 \mathrm{~h}$ at $37^{\circ} \mathrm{C}$. Caco-2/BBe cells were then exposed to 3HA-NHE3 adenovirus for $6 \mathrm{~h}$ at $37^{\circ} \mathrm{C}$. Cells were allowed to recover in normal media over the next $40 \mathrm{~h}$ before study $[28,29]$. 
shRNA knockdown (KD) of Cdc42

5 shRNA constructs for Cdc42 from Open Biosystems were obtained through the Johns Hopkins University School of Medicine High Throughput Biology Center (HiT Center). shRNA constructs were packaged into lentivirus particles using HEK293A cells. Caco-2/BBe cells were infected with shRNAcontaining lentiviruses and KD cells were selected using puromycin as a selection marker. Efficiency of KD was determined by Western blot. shRNA constructs that did not exhibit any measure of Cdc42 KD were used as negative controls.

\section{Measurement of $\mathrm{Na}^{+} / \mathrm{H}^{+}$Exchange}

Cellular $\mathrm{Na}^{+} / \mathrm{H}^{+}$exchange activity in Caco-2/BBe cells grown to 14-days post-confluency on Transwell filters was determined fluorometrically using the intracellular pH-sensitive dye, 2,7-bis(carboxyethyl)56-carboxyfluoresceinacetoxy-methy ester (BCECF- AM, $5 \mu \mathrm{M}$; Molecular Probes, Eugene, OR), as described previously [30]. Caco-2/BBe cells were exposed to $50 \mathrm{mM} \mathrm{NH}_{4} \mathrm{Cl}$ during a 45 -min dye loading, as described previously $[28,29,31]$. Cells were perfused initially with $\mathrm{TMA}^{+}$solution alone or with $10 \mu \mathrm{M}$ carbachol for 1-10min (138mM tetramethylammonium chloride, $5 \mathrm{mM} \mathrm{KCl}, 2 \mathrm{mM} \mathrm{CaCl}, 1 \mathrm{mM} \mathrm{MgSO}_{4}, 1 \mathrm{mM} \mathrm{NaH}_{2} \mathrm{PO}_{4}, 25$ mM glucose, $20 \mathrm{mM}$ HEPES, pH 7.4), before being switched to $\mathrm{Na}^{+}$solution (138mM NaCl instead of TMA ${ }^{+}$) for the $\mathrm{Na}^{+}$-dependent $\mathrm{pH}_{i}$ recovery. At the end of each experiment, the fluorescence ratio was calibrated to $\mathrm{pH}_{i}$ using the high potassium/nigericin method. $\mathrm{Na}^{+} / \mathrm{H}^{+}$exchange activity data were calculated as the ratio of $\mathrm{Na}^{+}$-dependent changes in $\mathrm{pH}_{i}$ over initial time $(\Delta \mathrm{pH} / \mathrm{min})$ of $\mathrm{Na}^{+}$-dependent $\mathrm{pH}$ recovery using at least three coverslips per condition in a single experiment. NHE3 activity was measured in cells treated with either vehicle or $\mathrm{CCH}(10 \mu \mathrm{M} ; 5 \mathrm{~min})$ in the presence or absence of $\mathrm{CPZ}(10 \mu \mathrm{M} ; 20 \mathrm{~min})$, methyl- $\beta$ cyclodextrin (M $\beta C D ; 10 \mathrm{mM} ; 20 \mathrm{~min})$, or Pirl-1 $(20 \mu \mathrm{M} ; 20 \mathrm{~min})$. NHE3 activity was measured in the presence

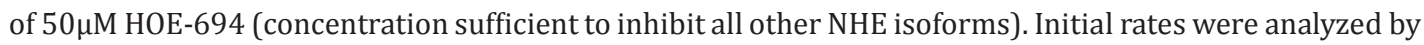
using Origin (Microcal Software) to determine statistical significance among individual experiments. Means \pm S.E. were determined from at least three separate experiments.

\section{Immunofluorescence}

Polarized Caco-2/BBe cells grown on Anapore filters were imaged under 4\% paraformaldahyde fixed conditions. NHE3 and clathrin colocalization was determined using standard confocal microscopy (Zeiss 510 META). NHE3 surface expression was observed using monoclonal anti-HA antibody conjugated to AlexaFluor 594 (1:100 dilution). The amount of surface NHE3 was compared against FITC-labeled wheat germ agglutinin (WGA) labeled Caco-2/BBe cell brush border (labeled at $4^{\circ} \mathrm{C}$ ).

\section{Co-immunoprecipitation}

Co-immunoprecipitation was performed as previously described [29]. Briefly, Caco-2/BBe cells were grown on $10-\mathrm{cm}^{2}$ Transwell Petri dishes until 12 days post-confluence. On day 12, cells were transduced with the adenovirus 3HA-NHE3 construct as described above. On day 14, cells were serum-starved for $4 \mathrm{~h}$ and treated either with vehicle or $10 \mu \mathrm{m} \mathrm{CCH}$ for $5 \mathrm{~min}$ at $37^{\circ} \mathrm{C}$. Cells were washed three times in ice-cold PBS containing $50 \mathrm{mM}$ Tris, scraped and lysed in $500 \mu$ l of ice-cold lysis buffer $(10 \mathrm{mM} \mathrm{HEPES}, 50 \mathrm{mM} \mathrm{NaCl}, 5$ mM EDTA, $1 \mathrm{mM}$ benzamidine, $0.5 \%$ Triton X-100). Cell lysates were incubated with either anti-CHC or anticaveolin-1 antibodies conjugated to Protein G-agarose beads (Sigma) for $2 \mathrm{~h}$ with end-over-end rotation at $4{ }^{\circ} \mathrm{C}$. Samples were washed five times with lysis buffer and immunoprecipitated proteins were eluted from beads with 2X sample buffer. Samples were resolved by 10\% SDS-PAGE and proteins were detected with anti-HA (for NHE3), anti-CHC and anti-caveolin antibodies and visualized on an Odyssey Infrared Imaging System (Li-Cor, Lincoln, NE). Results were obtained from three individual experiments.

\section{NHE3 surface biotinylation}

NHE3 surface biotinylation was performed as previously described [31]. Briefly, Caco-2/BBe cells (transiently expressing 3HA-tagged NHE3 adenovirus construct) monolayers were grown on Transwell filters (Corning). Cells treated with vehicle or CCH in the presence or absence of M $B C D$, CPZ or Pirl-1 were rinsed with ice-cold PBS and then incubated for 30min with gentle shaking in borate buffer (pH 8.0) with $1.25 \mathrm{mg} / \mathrm{ml} \mathrm{NHS}$-SS-biotin (Pierce). After rinsing with PBS, cells were washed twice for $10 \mathrm{~min}$ with $100 \mu \mathrm{M}$ glycine to scavenge unreacted biotin and scraped into lysis buffer with protease inhibitors. Lysates were incubated with avidin-agarose beads (Pierce) to precipitate the biotinylated proteins. The beads were then 
Fig. 1. Clathrin is not required for carbacholmediated inhibition of NHE3 Activity in Caco-/ BBe cells. NHE3 activity was measured by BCECF fluorimetry in differentiated Caco-2/BBe cells infected with adenovirus 3HA-NHE3 and exposed to either vehicle $(C T L=$ black bar $)$ or $10 \mu \mathrm{M}$ carbachol $(\mathrm{CCH})$ in the presence or absence of the clathrin inhibitor, chlorpromazine (CPZ; $10 \mu \mathrm{M})$. NHE3 activity was determined in the presence of $50 \mu \mathrm{M} \mathrm{HOE}$ 694 (concentration sufficient to inhibit all other NHE isoforms). $\mathrm{CCH}$ treatment resulted in a significant decrease of NHE3 activity in Caco-2/BBe/3HANHE3 cells in the presence and absence of CPZ for 30 minutes. In addition, CPZ treatment stimulated basal

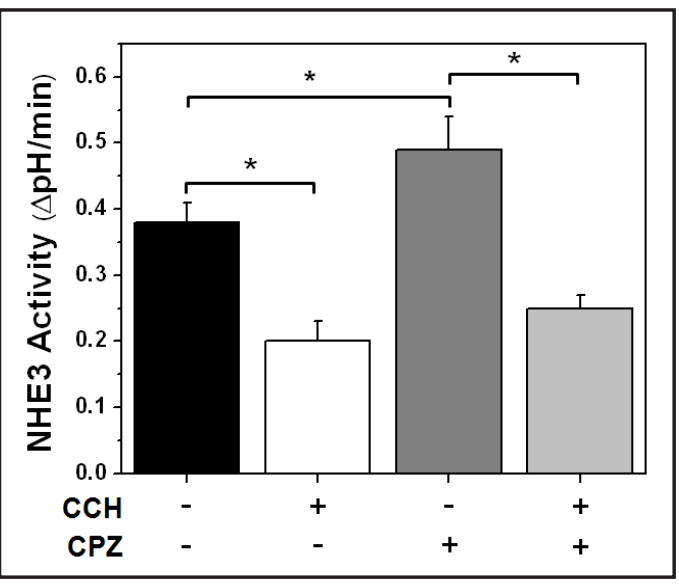

NHE3 activity in Caco-2/BBe cells by $\sim 29 \% . n \geq 4$ for each condition. Rate of NHE3 activity $(\Delta \mathrm{pH} / \mathrm{min})$ expressed as mean \pm S.E. ${ }^{*} p<0.05$ compared to untreated controls.

washed and biotinylated proteins separated by SDS-PAGE and transferred to nitrocellulose membranes for quantitative Western blotting (Odyssey Infrared Imaging System) using anti-HA (for NHE3) antibody. Surface biotinylated NHE3 was compared to total NHE3 protein levels.

Cdc42 actvation assay

Cell lysates were obtained from polarized Caco-2/BBe cells grown on Anapore filters for 14 days post-confluency. Caco-2/BBe cells were pretreated with vehicle or $35 \mu \mathrm{M}$ BAPTA-AM prior to exposure to $10 \mu \mathrm{M} \mathrm{CCH}$ for various time points (1-60min). Epidermal growth factor (EGF) is a potent activator of Cdc42 and was used as a positive control [32]. Cdc42 activity (i.e. bound to GTP) was measured using Cdc42 Activation Assay Biochem Kit (Cytoskeleton) according to the manufacture's protocol. Amount of activated Cdc42 was determined by Western blot analysis.

\section{Results}

Clathrin is not required for carbachol-mediated inhibition of NHE3 Activity in Caco-2/ BBe cells

Previous studies have demonstrated that constitutive NHE3 endocytosis occurs by a clathrin-dependent process $[17,23]$. Caco-2/BBe cells were grown on filters until postconfluent for 12 days and were then infected with an adenovirus 3HA-NHE3 construct. NHE3 activity was determined $48 \mathrm{~h}$ after the infection. Caco-2BBe/3HA-NHE3 cells were pretreated with either vehicle or $10 \mu \mathrm{M} \mathrm{CPZ}$ (blocks clathrin-coated pit formation) for $30 \mathrm{~min}$ and then exposed to vehicle or $\mathrm{CCH}$ for $5 \mathrm{~min}$, prior to $\mathrm{Na}^{+}$-dependent recovery of $\mathrm{pH}_{i}$ [33]. As shown in Figure 1, in vehicle pretreated controls, NHE3 activity was significantly inhibited $(\sim 47 \%)$ after $\mathrm{CCH}$ treatment (Fig. 1). In cells pretreated with CPZ, basal NHE3 activity was significantly increased by $29 \%$ compared to untreated controls $(p<0.05)$ while CCH inhibition of NHE3 activity was unaffected by CPZ pretreatment ( $49 \%)$. Similar results were obtained in cells exposed to $\mathrm{K}^{+}$-depleted conditions, which also blocks clathrin-mediated endocytosis by removing membrane-associated clathrin lattices (data not shown) [34]. These results suggest that basal NHE3 activity is, at least partially, regulated by clathrin-mediated endocytosis. However, CCH inhibition of NHE3 activity does not involve clathrin. Using immunofluorescence confocal microscopy, NHE3 and clathrin heavy chain did not appear to colocalize after $\mathrm{CCH}$ treatment but did exhibit some overlap under basal conditions (Fig. 2A). In order to demonstrate that CCH treatment alters the association of NHE3 and clathrin, we performed co-immunoprecipitation studies. As shown in Figure 2B, clathrin heavy chain (CHC) immunoprecipitated NHE3 under basal conditions and this association was reduced 


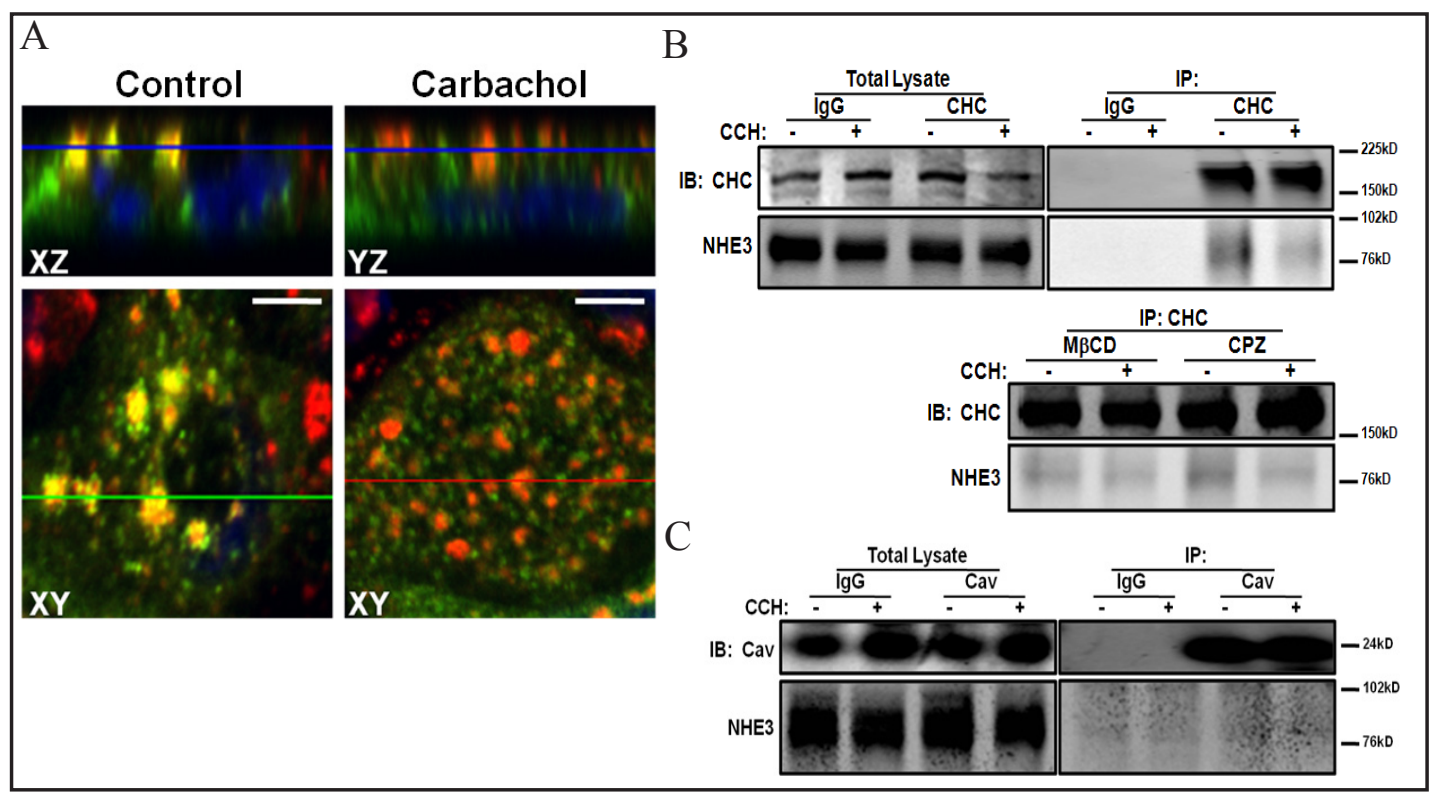

Fig. 2. Basal association of NHE3 with clathrin is reduced after carbachol treatment. $A$, NHE3 colocalization with clathrin heavy chain was determined by confocal microscopy in Caco-2/BBe cells treated with CCH for 5 minutes. After treatment, cells were fixed and stained with antibodies to label NHE3 and clathrin. Under basal conditions, NHE3 (green) and clathrin (red) demonstrated partial colocalization in the apical domain of Caco-2/BBe cells. After CCH treatment, NHE3 and clathrin did not exhibit any colocalization in any region of $\mathrm{Caco}-2 / \mathrm{BBe}$ cells. Scale bar $=5 \mu \mathrm{m}$. Images are representative of results from three independent experiments. $B$ - $C$, Association of NHE3 with clathrin and caveolin-1 was determined by coimmunoprecipitation (co-IP) studies in Caco-2/BBe cells treated with CCH for 5 min. Immunoprecipitated clathrin associates with NHE3 under basal conditions; however, this interaction is decreased after 5 min CCH treatment. Similar results were observed after CCH treatment in cells pretreated with CPZ or M $\beta$ CD. In contrast, NHE3 does not associate with caveolin-1 under any conditions in Caco-2/BBe cells. Rabbit polyclonal IgG antibody was used as a negative control. $n=3$ independent experiments for each co-IP study.

after 5 min $\mathrm{CCH}$ treatment. These results suggest that CCH-inhibition of NHE3 activity does not involve increased association with CHC. Furthermore, the association of NHE3 and CHC was not increased after $\mathrm{CCH}$ exposure in cells pretreated with $\mathrm{CPZ}$ or $\mathrm{M} \beta \mathrm{CD}$, which suggests that CCH inhibition of NHE3 activity involves a pool of NHE3 that does not associate with CHC. Moreover, we further demonstrated that caveolar-dependent endocytosis is also not involved in $\mathrm{CCH}$ regulation of NHE3 activity as immunoprecipiated caveolin-1 does not associate with NHE3 under basal or CCH treated conditions (Fig. 2C). Taken together, these results suggest that a non-clathrin, non-caveolar dependent pathway of endocytosis is involved in CCH-mediated inhibition of NHE3 activity. Previous studies have demonstrated that clathrin-independent endocytosis involves lipid rafts [21]. Furthermore, basal NHE3 activity is lipid raft dependent in OK cells [16] suggesting that an alternate pathway to clathrin-mediated endocytosis may exist for NHE3 trafficking. However, a role for lipid rafts in elevated $\left[\mathrm{Ca}^{2+}\right]$, regulation of NHE3 activity has not been demonstrated. We therefore tested whether constitutive and/or CCH- inhibition of NHE3 activity involves lipid rafts.

\section{Lipid rafts are necessary for CCH-inhibition of NHE3 activity}

We determined the role of lipid rafts in basal and CCH inhibition of NHE3 activity using the cholesterol depleting agent, methyl- $\beta$-cylcodextrin (M $\beta C D$ ). In Caco-2/BBe cells pretreated with $\mathrm{M} \beta \mathrm{CD}$, basal NHE3 activity was stimulated by $30 \%$ (Fig. 3). This result suggests that a portion of BB NHE3 is associated with lipid rafts and that this fraction 


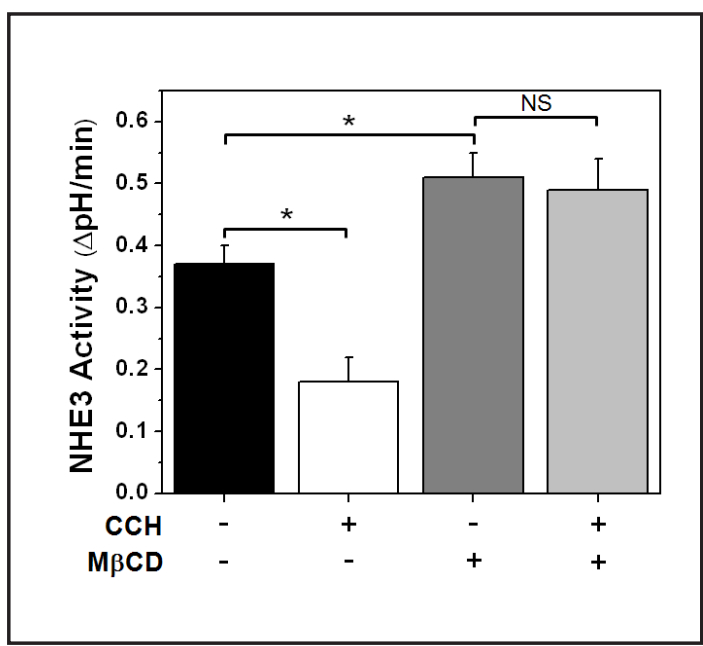

Fig. 3. Lipid rafts are necessary for carbacholinhibition of NHE3 activity. NHE3 activity was measured in Caco-2/BBe/3HA-NHE3 exposed to either vehicle $(C T L=$ black bar $)$ or $10 \mu \mathrm{M}$ carbachol $(\mathrm{CCH})$ in the presence or absence of $10 \mathrm{mM}$ methyl$\beta$-cyclodextrin $(M \beta C D)$ pretreated for $20 \mathrm{~min}$. NHE3 activity was determined in the presence of 50 $\mu \mathrm{M}$ HOE-694 (concentration sufficient to inhibit all other NHE isoforms). $\mathrm{CCH}$ treatment resulted in a significant decrease of NHE3 activity in Caco2/BBe/3HA-NHE3 cells. In contrast, inhibition of NHE3 activity by $\mathrm{CCH}$ was completely abolished in Caco-2/BBe/3HA-NHE3 cells exposed to M $\beta C D$. $\mathrm{M} \beta \mathrm{CD}$ also significantly stimulated basal NHE3 activity in Caco-2/BBe cells by $\sim 30 \%$. $n \geq 5$ for each condition. Rate of NHE3 activity $(\Delta \mathrm{pH} / \mathrm{min})$ expressed as mean \pm S.E. ${ }^{*} p<0.05$ compared to untreated controls. NS $=$ Not significant.

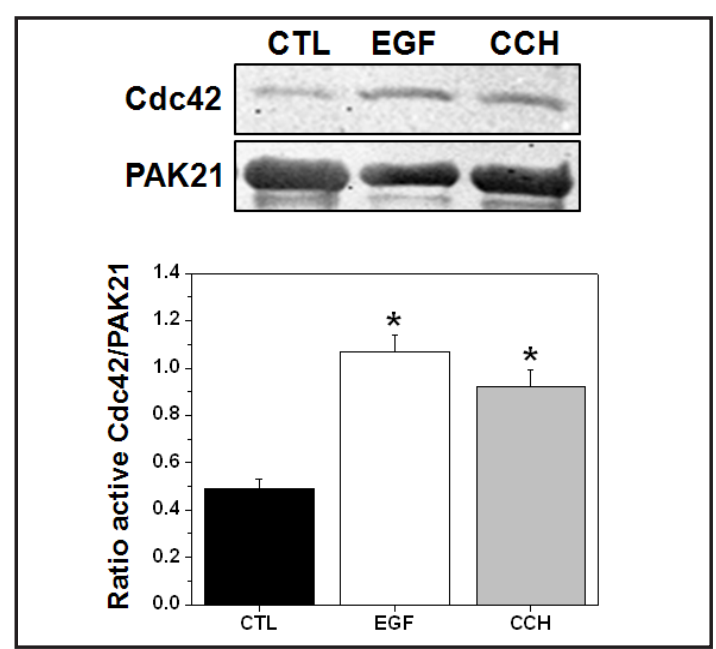

Fig. 4. Carbachol signaling increases the amount of GTP-bound Cdc42 in Caco-2/BBe cells. The amount of GTP-bound Cdc42 under basal (CTL) and carbachol $(\mathrm{CCH})$ treated conditions in Caco-2/ BBe cells was measured by pull-down assay using purified PAK21 binding protein. Caco-2/BBe cells were untreated or treated with $\mathrm{CCH}$ for $5 \mathrm{~min}$, cells washed in ice cold PBS, and cell lysates collected. GTP-bound Cdc42 (i.e. activated Cdc42) was precipitated from cell lysates with PAK21 binding protein and visualized by western blot. $10 \mu \mathrm{M}$ CCH treatment significantly increased the amount of GTP-bound Cdc42 compared to controls. Treatment of Caco-2/BBe cells with EGF served as a positive control. Blots represent results obtained from three independent experiments. Cdc42 protein levels normalized to PAK21 expressed as mean \pm S.E. ${ }^{*} p<0.05$ compared to controls.

participates in constitutive endocytosis since disruption of lipid rafts results in increased NHE3 activity. This result is supported by surface biotinylation studies where BB NHE3 levels are increased by $\sim 24 \%$ after CCH exposure in M $\beta C D$-treated cells (data not shown). In contrast to pretreatment with $\mathrm{CPZ}$, disruption of lipid rafts by $\mathrm{M} \beta \mathrm{CD}$ treatment completely abolished CCH inhibition of NHE3 activity (Fig. 3). Our current results in Caco-2/BBe cells support previous studies which demonstrated that a pool of BB NHE3 resides in lipid rafts in intestinal epithelial cells. Furthermore, we demonstrate that $\mathrm{M} \beta \mathrm{CD}$ treatment completely prevented CCH inhibition of NHE3 activity suggesting that the pool of BB NHE3 inhibited by $\mathrm{CCH}$ is in lipid rafts (Fig. 3). Since all clathrin-independent endocytosis has been shown to involve lipid rafts and depend on small GTPases, we next tested whether $\mathrm{CCH}$ inhibition of NHE3 activity required the Rho GTPase, Cdc42 [35].

\section{Cdc42 is necessary for CCH-inhibition of NHE3 activity}

Previous studies demonstrated that clathrin-independent endocytosisinvolves members of the Rho family of small GTPases [36]. Examples of clathrin-independent endocytosis that are dependent on small GTPases include CLIC/GEEC and macropinocytosis [21]. Since Cdc42 is required for some types of clathrin-independent endocytosis, we tested whether basal or $\mathrm{CCH}$ inhibition of NHE3 activity required Cdc42. In order to demonstrate that $\mathrm{CCH}$ signaling 
Fig. 5. Cdc42 is necessary for carbachol-inhibition of NHE3 activity. NHE3 activity was measured in Caco2/BBe/3HA-NHE3 exposed to either vehicle $(C T L=$ black bar) or $10 \mu \mathrm{M}$ carbachol $(\mathrm{CCH})$ in the presence or absence of the Cdc42 specific inhibitor, Pirl-1 $(20 \mu \mathrm{M}$; pretreated for $30 \mathrm{~min}$. NHE3 activity was determined in the presence of $50 \mu \mathrm{M}$ HOE-694 (concentration sufficient to inhibit all other NHE isoforms). $\mathrm{CCH}$ treatment resulted in a significant decrease of NHE3 activity in Caco-2/BBe/3HA-NHE3 cells. In contrast, inhibition of NHE3 activity by $\mathrm{CCH}$ was completely abolished in Caco-2/BBe/3HA-

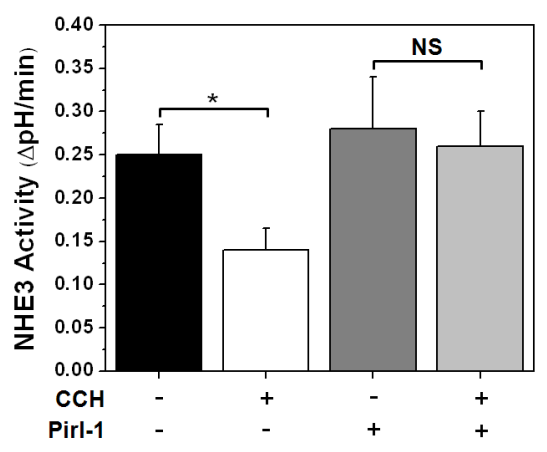

NHE3 cells exposed to Pirl-1. Exposure of cells to Pirl-1 did not alter basal NHE3 activity in Caco-2/BBe cells. $n \geq 3$ for each condition. Rate of NHE3 activity ( $\Delta \mathrm{pH} / \mathrm{min}$ ) expressed as mean \pm S.E. ${ }^{*} p<0.05$ compared to untreated controls. NS = Not significant.

Fig. 6. shRNA knockdown (KD) of Cdc42 in Caco-2/ BBe cells prevents carbachol inhibition of NHE3 activity. Western blot analysis of total cell lysates prepared from Caco2/BBe cells stably transduced with lentivirus empty vector (EV) control or Cdc42 (left panel) shRNA constructs. Two different shRNA constructs (out of 5 total) are shown. shRNA A for Cdc42 represents the

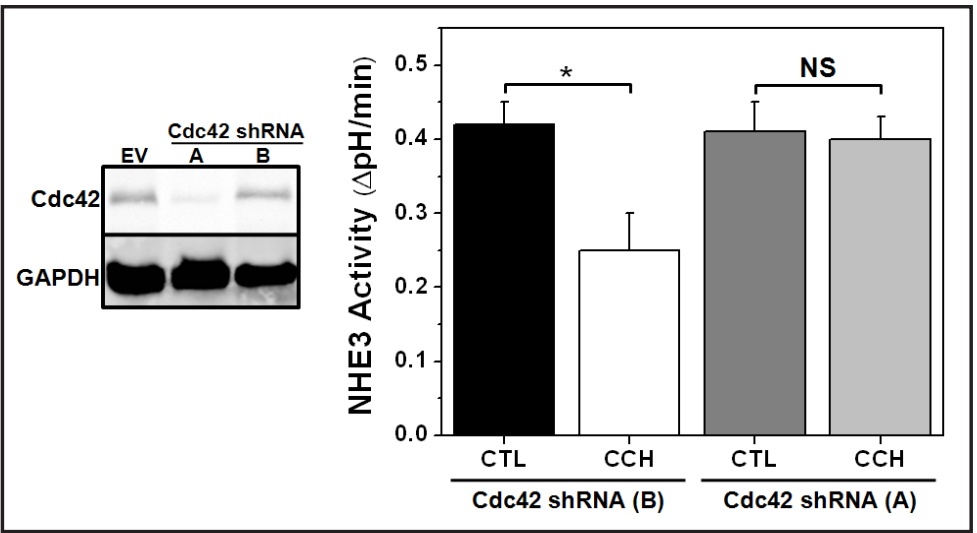
construct that demonstrated greatest degree of KD (84\% compared to negative controls). shRNA B for Cdc42 did not exhibit any degree of KD compared to EV controls. Glyceraldehyde-3-phosphate dehydrogenase $(G A P D H)$ was used as internal control. Stable Cdc42 KD Caco-2/BBe cells were treated with vehicle (CTL) or $10 \mu \mathrm{M}$ carbachol $(\mathrm{CCH})$ for $5 \mathrm{~min}$. NHE3 activity was determined in the presence of $50 \mu \mathrm{M}$ HOE-694 (concentration sufficient to inhibit all other NHE isoforms). CCH treatment resulted in a significant decrease of NHE3 activity in Cdc42 KD shRNA B cells but this effect was lost in Cdc42 KD shRNA A cells. Basal NHE3 activity in each line was not affected. $n \geq 3$ for each condition. Rate of NHE3 activity $(\Delta \mathrm{pH} / \mathrm{min})$ expressed as mean \pm S.E. ${ }^{*} p<0.05$ compared to untreated controls. NS $=$ Not significant.

activates Cdc42, we measured the amount of GTP-bound Cdc42 (i.e. active form) after CCH treatment. After 5min CCH treatment, the levels of GTP-bound Cdc42 were significantly increased compared to untreated controls (Fig. 4). In fact, Cdc42 activation occurred as early as $1 \mathrm{~min}$ post $\mathrm{CCH}$ and remained elevated for up to $20 \mathrm{~min}$ (data not shown). We then pretreated Caco-2/BBe cells with the Cdc42 specific inhibitor, Pirl-1, and measured its effect on NHE3 activity. As shown in Figure 5, Pirl-1 treatment ( $20 \mu \mathrm{M}$ for $30 \mathrm{~min})$ prevented CCH inhibition of NHE3 activity, but did not affect basal activity. We further demonstrated a role for Cdc42 in CCH regulation using shRNA knockdown technology. We tested multiple shRNA probes and generated stable Cdc42 KD Caco-2/BBe cells (Fig. 6). By Western blot, Cdc42 KD (denoted as Cdc42 shRNA A) reduced expression by 74\% compared to empty vector and ineffective Cdc42 shRNA constructs (Cdc42 shRNA B; Fig. 6). We then tested the effect of Cdc42 KD on NHE3 under basal and CCH treated conditions. As demonstrated in Figure 6, Cdc42 KD abolished CCH regulation of NHE3 activity but did not alter basal NHE3 activity 
Fig. 7. Inhibition of Cdc42, but not clathrin, prevent carbachol-induced endocytosis of NHE3 in Caco-2/BBe cells. Caco-2/BBe cells infected with 3HA-NHE3 adenovirus construct were treated with either vehicle (control) or $10 \mu \mathrm{M}$ carbachol $(\mathrm{CCH})$ for $5 \mathrm{~min}$ after pretreatment with either Pirl-1 or CPZ. After vehicle or $\mathrm{CCH}$ exposure, cells were fixed in $4 \%$ paraformaldehyde in PBS, permeabilized and stained for NHE3 (red; anti-HA antibody) and wheat germ aglutinnin (green; AlexaFluor 488 conjugated WGA) to mark BB. XY views are shown. $A$, NHE3 and WGA colocalize in the BB of untreated control cells. $B$, NHE3 co-localization with WGA and expression in the $\mathrm{BB}$ was reduced after $\mathrm{CCH}$ treatment. NHE3 appeared in intracellular vesicles below BB (arrowheads). C, Pretreatment of cells with Pirl1 prevented $\mathrm{CCH}$ induced endocytosis of NHE3 while in $D, \mathrm{CPZ}$ treatment had no effect on the $\mathrm{CCH}$ induced changes in NHE3 localization and displayed similar localization to that in $B$. Scale bar $=20 \mu \mathrm{m}$. Results were similar in 4 independent experiments. $E$, The effect of CPZ and Pirl-1 on CCH-mediated endocytosis of NHE3 was determined by surface biotinylation studies. Caco-2/BBe cells were treated with vehicle or $\mathrm{CCH}$ in the presence or absence of

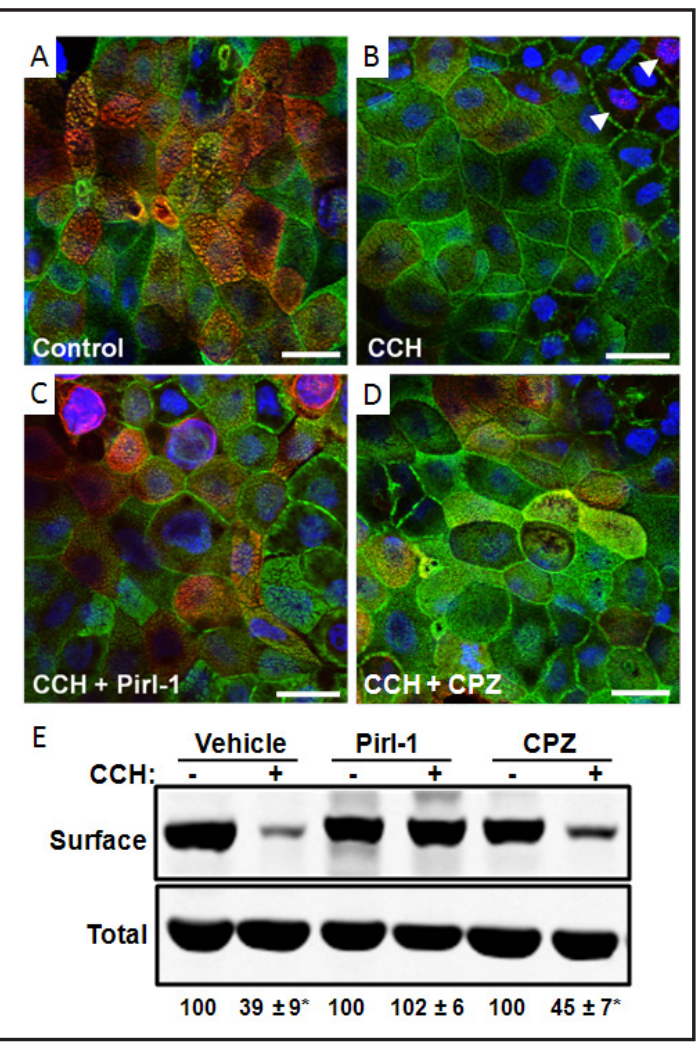
CPZ or Pirl-1 and surface biotinylated NHE3 was detected by Western blot. CCH decreased BB NHE3 levels, an effect that was lost when cells were pretreated with Pirl-1, but not CPZ. Surface NHE3 was normalized to total NHE3 in each treatment group and expressed as percent control. Numbers indicate percent change after $\mathrm{CCH}$ treatment compared to respective controls. Results were similar in five independent experiments. ${ }^{*} p<0.05$ compared to controls.

when compared to Cdc42 shRNA KD B cells. These data support the hypothesis that CCH induced endocytosis of NHE3 requires lipid rafts and Cdc42.

Inhibition of Cdc42, but not clathrin, prevents CCH-induced endocytosis of NHE3 in Caco-2/BBe cells

Since it is well established that CCH inhibition of NHE3 occurs by increased endocytosis, we performed immunofluorescence confocal microscopy to determine the localization of NHE3 under basal and CCH conditions. As shown in Figure 7A, NHE3 was localized to the BB of Caco-2/BBe cells similar to the localization of the BB marker, WGA, under basal conditions. After CCH treatment (Fig. 7B), the amount of NHE3 in the BB was reduced compared to WGA. Also, intracellular NHE3 vesicles (arrowheads) were observed in an area above the nucleus but below the BB after $\mathrm{CCH}$ treatment. We further demonstrated whether inhibition of clathrin or Cdc42 prevented CCH induced internalization of NHE3. In cells pretreated with Pirl-1, NHE3 colocalized with WGA in the BB indicating a block in endocytosis after $\mathrm{CCH}$ treatment (Fig. 7C). In contrast, NHE3 and WGA colocalization was lost in cells pretreated with chlorpromazine demonstrating that CCH-induced endocytosis of NHE3 occurred even though clathrin-mediated endocytosis was inhibited (Fig. 7D). In order to confirm that changes in BB NHE3 localization under these conditions, we performed surface biotinylation studies. In Caco-2/BBe cells treated with $\mathrm{CCH}$, surface NHE3 expression was decreased by $\sim 60 \%$ (Fig. 7E). This effect was lost in CCH treated cells pretreated with Pirl1, suggesting that CCH-induced endocytosis of NHE3 involves Cdc42. Furthermore, CPZ 
Fig. 8. Carbachol activation of Cdc42 is calcium dependent. Caco-2/BBe cells were pretreated with vehicle $(C T L)$ or $35 \mu \mathrm{M}$ BAPTA-AM $(B A P)$ for 30 minutes prior to treatment with carbachol $(\mathrm{CCH})$ for $5 \mathrm{~min}$. Cells lysates from each condition were obtained and the amount of GTP-bound Cdc42 was quantified by Western blot analysis. CCH treatment increased the amount of GTP-bound Cdc42 compared to controls, an effect that was prevented by BAPTA-AM pretreatment. Blots are representative of results obtained from three independent experiments. Cdc42 protein levels normalized to PAK21 expressed as mean \pm S.E. $* p<0.05$ compared to controls.

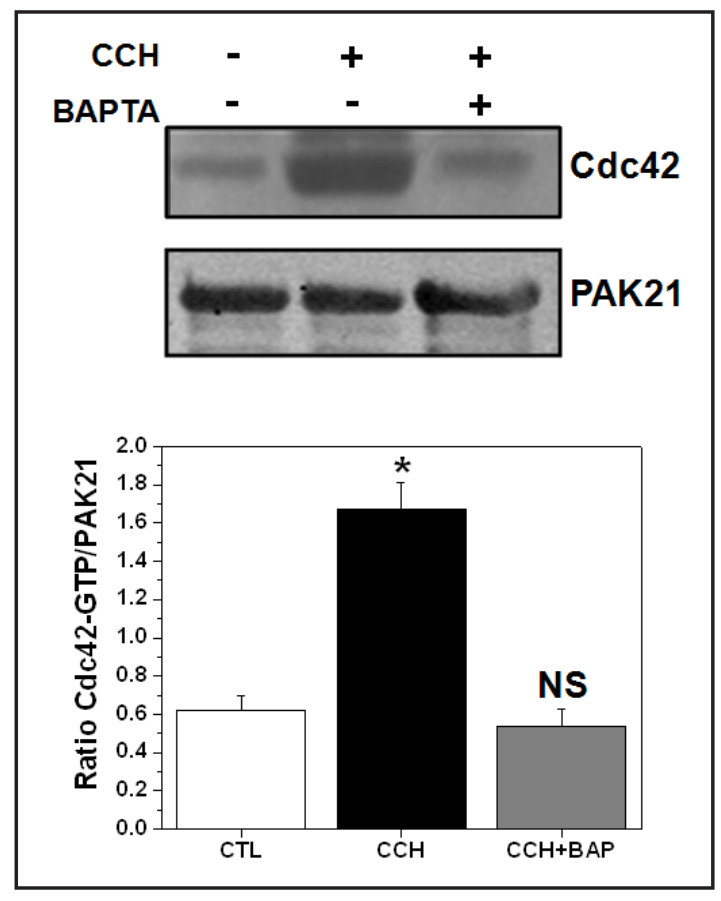

treatment did not prevent CCH-mediated internalization of NHE3 further demonstrating that CME is not involved (Fig. 7E). These results also demonstrate that $\mathrm{CCH}$ activation of Cdc42 is necessary for clathrin-independent endocytosis of NHE3 in Caco-2/BBe cells. Since $\mathrm{CCH}$ signaling involves elevated $\left[\mathrm{Ca}^{2+}\right]_{\mathrm{i}}$, we next determined whether activation of Cdc42 by $\mathrm{CCH}$ was calcium dependent.

\section{CCH-induced activation of Cdc42 is $\mathrm{Ca}^{2+}$-dependent}

In order to demonstrate that $\mathrm{CCH}$ activation of $\mathrm{Cdc} 42$ depends on elevated $\left[\mathrm{Ca}^{2+}\right]_{\mathrm{i}}$, we measured the levels of GTP-bound Cdc42 in Caco-2/BBe cells pretreated with the $\left[\mathrm{Ca}^{2+}\right]_{\mathrm{i}}$ chelator, BAPTA-AM $(35 \mu \mathrm{M})$ prior to $\mathrm{CCH}$ treatment. As shown in Figure 8, CCH treatment (5min) increased the amount of GTP-bound Cdc42 compared to control conditions. Pretreatment with BAPTA-AM prevented $\mathrm{CCH}$-induced increase in GTP-bound Cdc42 suggesting that $\mathrm{CCH}$-induced increase in GTP-bound Cdc42 levels depend on elevation of $\left[\mathrm{Ca}^{2+}\right]_{i}$. Taken together, the results of the current study demonstrate that $\mathrm{CCH}$ inhibition of NHE3 activity is due to increased endocytosis by a clathrin-independent mechanism that requires lipid rafts and Cdc42 in Caco-2/BBe cells.

\section{Discussion}

This study provides additional insights into the trafficking mechanism involved in regulation of intestinal $\mathrm{Na}^{+}$absorption under elevated $\left[\mathrm{Ca}^{2+}\right]_{i}$ conditions. NHE3 is regulated by changes in its plasma membrane versus intracellular location as a result of alterations in the rates of endocytosis and/or exocytosis. NHE3 trafficking occurs under basal and acutely inhibited conditions (cAMP, cGMP, elevated $\left[\mathrm{Ca}^{2+}\right]_{\mathrm{i}}$ ) [1]. Moreover, previous studies have observed that constitutive and cAMP stimulated NHE3 endocytosis occur through a clathrin-dependent mechanism [17]. However, the mechanisms responsible for NHE3 trafficking in intestinal epithelial cells are not well understood. Recently, studies using confocal immunofluorescence microscopy and transmission electron microscopy (TEM) have characterized nine clathrin-independent endocytosis pathways in addition to clathrin-mediated endocytosis (CME) [21]. While previous studies have demonstrated that constitutive NHE3 endocytosis occurs via CME, our data demonstrates a role for clathrin- 
independent endocytosis in elevated $\left[\mathrm{Ca}^{2+}\right]_{\mathrm{i}}$ regulation of NHE3 activity. Our concept that NHE3 endocytosis may occur through multiple endocytic pathways is novel and our current study is the first to separate NHE3 endocytosis into clathrin-dependent and clathrinindependent pathways.

Recently, Musch et al demonstrated that synaptotagmin 1 regulates cAMP and $\mathrm{Ca}^{2+}$ mediated inhibition of NHE3 activity in a clathrin-dependent manner [17]. In their study, the effect of elevated $\left[\mathrm{Ca}^{2+}\right]_{\mathrm{i}}$ on NHE3 trafficking was determined using thapsigargin, which was used to elevate cytoplasmic $\mathrm{Ca}^{2+}$ by preventing reuptake of released $\mathrm{Ca}^{2+}$ into intracellular stores. This study concluded that $\mathrm{Ca}^{2+}$ stimulated endocytosis of NHE3 occurred in a similar manner to that of cAMP. While this study evaluated the downstream effect of elevated $\left[\mathrm{Ca}^{2+}\right]_{i}$ in stimulating NHE3 endocytosis, the study did not determine whether ligand-receptor mediated $\mathrm{Ca}^{2+}$ signaling regulates NHE3 endocytosis in a similar manner. We have previously demonstrated in the intact small intestine that carbachol (CCH) signaling, which elevates apical $\left[\mathrm{Ca}^{2+}\right]_{i}$ via basolateral membrane (BLM) muscarinic receptors (M3), inhibited NHE3 activity by stimulating endocytosis [2-5, 14]. However, elevation of $\left[\mathrm{Ca}^{2+}\right]_{\mathrm{i}}$ using the calcium ionophore, A23187, alone was not able to duplicate all of the effects of BLM M3 cholinergic signaling [37]. These results emphasize the importance of using physiologically relevant agents to study the mechanisms responsible for $\mathrm{Ca}^{2+}$ inhibition of NHE3 activity in intestinal epithelial cells.

The results from the current study are the first to demonstrate that $\mathrm{CCH}$ inhibition of NHE3 activity is entirely lipid raft dependent. NHE3 exists in multiple pools in the BB of intestinal and renal epithelial cells; however, the regulation of NHE3 in these different pools has only been partially described $[15,16]$. In the current study, we demonstrate that basal NHE3 activity is increased after M $\beta C D$ treatment in Caco-2/BBe cells (Fig. 3). These results suggest that a pool of NHE3 resides in lipid rafts under basal conditions and participates in constitutive recycling. A role for lipid rafts has been suggested in both intestinal and renal epithelial cells; although, NHE3 is not believed to undergo stimulated endocytosis in renal proximal tubule cells [6, 38-41]. In the intestine, $\mathrm{CCH}$ stimulates rapid translocation of signaling proteins to detergent resistant membrane fractions that include lipid rafts [13]. In fact, pretreatment of the rabbit ileum with $\mathrm{M} \beta \mathrm{CD}$ prior to $\mathrm{CCH}$, prevented the increase in the size of NHE3 containing multi-protein complexes that result from CCH signaling [13]. Based on these findings, it is possible that disruption of lipid rafts may prevent organization of signaling complexes that are required for $\mathrm{CCH}$-mediated endocytosis. However, the mechanism by which lipid rafts contribute to inhibition of NHE3 activity remains to be determined.

It has been recently demonstrated that clathrin-independent endocytosis requires lipid rafts while clathrin-mediated endocytosis does not. Since several types of clathrinindependent endocytosis pathways have been described, the goal of the current study was to separate the contributions of clathrin-dependent and clathrin-independent endocytosis to basal and $\mathrm{CCH}$ inhibition of NHE3 activity. Our results show that basal, but not CCHinhibited, NHE3 activity involves clathrin-mediated endocytosis based on studies using chlorpromazine and $\mathrm{K}^{+}$-depletion to prevent CME. Although all inhibitors of CME have been shown to elicit non-specific effects on trafficking, our results support previous studies that constitutive NHE3 endocytosis is clathrin-dependent [17, 23]. Whether additional CME inhibitors affect basal NHE3 trafficking in a similar manner to CPZ and $\mathrm{K}^{+}$-depletion remains to be determined. Our results also demonstrate a modest $(\sim 30 \%)$ increase in basal NHE3 activity after $\mathrm{M} \beta \mathrm{CD}$ treatment suggesting that a portion of $\mathrm{BB}$ NHE3 resides in lipid rafts under basal conditions and is inhibitory of basal NHE3 activity in Caco-2/BBe cells. These data also support previous studies in the intact rabbit ileum and in renal proximal tubule cell lines that demonstrate a role for lipid rafts in regulating basal NHE3 activity $[15,16]$. However, under elevated $\left[\mathrm{Ca}^{2+}\right]$ conditions, NHE3 regulation is completely lipid raft dependent. These results contrast with previous studies in which 
calcium ionophores or thapsigargin were used to elevate $\left[\mathrm{Ca}^{2+}\right]_{i}$. In these studies, CME was responsible for NHE3 endocytosis. In this study, we administered $\mathrm{CCH}$ to mimic the neurohumoral regulation of normal digestion. $\mathrm{CCH}$ binds to basolateral muscarinic (M3) receptors to elicit their physiological effects. Recent studies have linked CCH signaling to stimulation of clathrin-independent endocytosis; however, more detailed studies of the signaling molecules involved in this pathway are necessary [42].

Several types of clathrin-independent endocytosis have been described to require small GTPases including Cdc42, which has been shown to play an integral role in CLIC/ GEEC and macropinocytosis [21]. Our results are the first to demonstrate a role for Cdc42 in NHE3 regulation. Here we show that CCH inhibition of NHE3 activity is also entirely Cdc42 dependent. Furthermore, we demonstrate that activated Cdc42 is required for $\mathrm{CCH}$ inhibition of NHE3 activity and that activation of Cdc42 occurs rapidly ( $\sim 1 \mathrm{~min})$ after $\mathrm{CCH}$ treatment. This rapid signaling is consistent with previous studies demonstrating rapid activation of other $\mathrm{CCH}$ signaling events including activated PLC- $\gamma$ and PKC at the apical membrane in intestinal epithelial cells. Cdc42 is well established to play an integral role in actin remodeling events that are necessary for endocytic vesicle formation and vesicle trafficking. These results in combination with our lipid raftresults suggest that $\mathrm{CCH}$-induced endocytosis of NHE3 involves a clathrin-independent pathway. However, identification of the type of clathin-independent pathway involved is not known. Furthermore, more detailed studies about the role of $\mathrm{Cdc} 42$ in actin remodeling under $\mathrm{CCH}$ treated conditions are necessary to characterize the type of clathrin-independent endocytosis that occurs under these conditions.

Why does NHE3 traffic through clathrin-dependent and clathrin-independent pathways? Recent studies have suggested that membrane cargo can internalize through multiple endocytic pathways [43]. These studies also demonstrated that different endocytic pathways (i.e. clathrin-dependent versus clathrin-independent) may lead to separate intracellular destinations including recycling endosomes versus lysosomes/proteosomes [44]. Other studies have demonstrated that different membrane cargo internalized by either clathrin-dependent or clathrin-independent pathways can merge and share similar intracellular endocytic compartments, including early and recycling endosomes [43]. It has been shown that CCH treatment results in the increased association of NHE3 with EEA1-positive endosomes [13]. A recent report has observed that clathrin-independent endocytosis directs MHC-I trafficking through EEA1 endosomes and eventually leads to delivery to lysosomes for degradation [38]. Whether NHE3 protein expression is downregulated in response to CCH treatment or whether other NHE3 trafficking involves alternate endosomal compartments remains to be determined.

The results of this study demonstrate that CCH inhibition of NHE3 activity requires lipid rafts and activated Cdc42 and suggests that NHE3 inhibition involves a clathrin-independent endocytosis pathway. Whether this pathway is similar to a clathrin-independent pathway that has been previously described or represents a novel variation remains to be determined. Furthermore, we demonstrate that NHE3 regulation by endocytosis occurs through multiple pathways and suggests a higher order of second messenger regulation than previously recognized. The results of our study may provide insight into how $\mathrm{Na}^{+}$absorption is regulated under the elevated calcium conditions observed under normal digestion and in some acute diarrheal diseases.

\section{Acknowledgements}

The Johns Hopkins Ross Confocal Facility and The Kudsi Imaging Core of the Hopkins Conte Digestive Disease Basic and Translational Research Core Center. This project was funded by the NIH: K01-DK080930 and R03-DK091482 


\section{References}

1 Zachos NC, Tse M, Donowitz M: Molecular physiology of intestinal $\mathrm{Na}^{+} / \mathrm{H}^{+}$exchange. Annu Rev Physiol 2005;67:411-443.

2 Cohen ME, Wesolek J, McCullen J, Rys-Sikora K, Pandol S, Rood RP, Sharp GW, Donowitz M: Carbachol- and elevated $\mathrm{Ca}^{2+}$-induced translocation of functionally active protein kinase $\mathrm{c}$ to the brush border of rabbit ileal $\mathrm{Na}^{+}$absorbing cells. J Clin Invest 1991;88:855-863.

3 Donowitz M, Cohen ME, Gould M, Sharp GW: Elevated intracellular $\mathrm{Ca}^{2+}$ acts through protein kinase $\mathrm{c}$ to regulate rabbit ileal nacl absorption. Evidence for sequential control by $\mathrm{Ca}^{2+} /$ calmodulin and protein kinase c. J Clin Invest 1989;83:1953-1962.

4 Kasai $\mathrm{H}$, Augustine GJ: Cytosolic $\mathrm{Ca}^{2+}$ gradients triggering unidirectional fluid secretion from exocrine pancreas. Nature 1990;348:735-738.

5 Tapper EJ, Powell DW, Morris SM: Cholinergic-adrenergic interactions on intestinal ion transport. Am J Physiol 1978;235:E402-409.

6 Bobulescu IA, Dwarakanath V, Zou L, Zhang J, Baum M, Moe OW: Glucocorticoids acutely increase cell surface $\mathrm{Na}^{+} / \mathrm{H}^{+}$exchanger-3 (nhe3) by activation of nhe3 exocytosis. Am J Physiol Renal Physiol 2005;289:F685-691.

7 Collazo R, Fan L, Hu MC, Zhao H, Wiederkehr MR, Moe OW: Acute regulation of $\mathrm{Na}^{+} / \mathrm{H}^{+}$exchanger nhe3 by parathyroid hormone via nhe3 phosphorylation and dynamin-dependent endocytosis. J Biol Chem 2000;275:31601-31608.

8 Donowitz M, Janecki A, Akhter S, Cavet ME, Sanchez F, Lamprecht G, Zizak M, Kwon WL, Khurana S, Yun CH, Tse CM: Short-term regulation of nhe3 by egf and protein kinase $\mathrm{c}$ but not protein kinase a involves vesicle trafficking in epithelial cells and fibroblasts. Ann N Y Acad Sci 2000;915:30-42.

-9 Hu MC, Fan L, Crowder LA, Karim-Jimenez Z, Murer H, Moe OW: Dopamine acutely stimulates $\mathrm{Na}^{+} / \mathrm{H}^{+}$ exchanger (nhe3) endocytosis via clathrin-coated vesicles: Dependence on protein kinase a-mediated nhe3 phosphorylation. J Biol Chem 2001;276:26906-26915.

10 Janecki AJ, Montrose MH, Zimniak P, Zweibaum A, Tse CM, Khurana S, Donowitz M: Subcellular redistribution is involved in acute regulation of the brush border $\mathrm{Na}^{+} / \mathrm{H}^{+}$exchanger isoform 3 in human colon adenocarcinoma cell line caco-2. Protein kinase c-mediated inhibition of the exchanger. J Biol Chem 1998;273:8790-8798.

11 Li X, Zhang H, Cheong A, Leu S, Chen Y, Elowsky CG, Donowitz M: Carbachol regulation of rabbit ileal brush border $\mathrm{Na}^{+} / \mathrm{H}^{+}$exchanger 3 (nhe3) occurs through changes in nhe3 trafficking and complex formation and is src dependent. J Physiol 2004;556:791-804.

12 Moe OW: Acute regulation of proximal tubule apical membrane na/h exchanger nhe-3: Role of phosphorylation, protein trafficking, and regulatory factors. J Am Soc Nephrol 1999;10:2412-2425.

13 Orlowski J, Grinstein S: $\mathrm{Na}^{+} / \mathrm{H}^{+}$exchangers of mammalian cells. J Biol Chem 1997;272:22373-22376.

14 Zachos NC, Kovbasnjuk O, Donowitz M: Regulation of intestinal electroneutral sodium absorption and the brush border $\mathrm{Na}^{+} / \mathrm{H}^{+}$exchanger by intracellular calcium. Ann N Y Acad Sci 2009;1165:240-248.

15 Li X, Galli T, Leu S, Wade JB, Weinman EJ, Leung G, Cheong A, Louvard D, Donowitz M: $\mathrm{Na}^{+} / \mathrm{H}^{+}$exchanger 3 (nhe3) is present in lipid rafts in the rabbit ileal brush border: A role for rafts in trafficking and rapid stimulation of nhe3. J Physiol 2001;537:537-552.

16 Murtazina R, Kovbasnjuk O, Donowitz M, Li X: Na ${ }^{+} / \mathrm{H}^{+}$exchanger nhe3 activity and trafficking are lipid raftdependent. J Biol Chem 2006;281:17845-17855.

17 Musch MW, Arvans DL, Walsh-Reitz MM, Uchiyama K, Fukuda M, Chang EB: Synaptotagmin i binds intestinal epithelial nhe3 and mediates camp- and $\mathrm{Ca}^{2+}$-induced endocytosis by recruitment of ap2 and clathrin. Am J Physiol Gastrointest Liver Physiol 2007;292:G1549-1558.

18 Chadda R, Howes MT, Plowman SJ, Hancock JF, Parton RG, Mayor S: Cholesterol-sensitive cdc42 activation regulates actin polymerization for endocytosis via the geec pathway. Traffic 2007;8:702-717.

19 Gauthier NC, Monzo P, Kaddai V, Doye A, Ricci V, Boquet P: Helicobacter pylori vaca cytotoxin: A probe for a clathrin-independent and cdc42-dependent pinocytic pathway routed to late endosomes. Mol Biol Cell 2005; 16:4852-4866.

20 Haigler HT, McKanna JA, Cohen S: Rapid stimulation of pinocytosis in human carcinoma cells a-431 by epidermal growth factor. J Cell Biol 1979;83:82-90.

-21 Doherty GJ, McMahon HT: Mechanisms of endocytosis. Annu Rev Biochem 2009;78:857-902. 


\section{Cellular Physiology Cell Physiol Biochem 2014;33:869-881 and Biochemistry

22 Lundmark R, Doherty GJ, Howes MT, Cortese K, Vallis Y, Parton RG, McMahon HT: The gtpase-activating protein graf1 regulates the clic/geec endocytic pathway. Curr Biol 2008;18:1802-1808.

23 Chow CW, Khurana S, Woodside M, Grinstein S, Orlowski J: The epithelial $\mathrm{Na}^{+} / \mathrm{H}^{+}$exchanger, nhe3, is internalized through a clathrin-mediated pathway. J Biol Chem 1999;274:37551-37558.

24 Kim JH, Lee-Kwon W, Park JB, Ryu SH, Yun CH, Donowitz M: $\mathrm{Ca}^{2+}$-dependent inhibition of $\mathrm{Na}^{+} / \mathrm{H}^{+}$exchanger 3 (nhe3) requires an nhe3-e3karp-alpha-actinin-4 complex for oligomerization and endocytosis. J Biol Chem 2002;277:23714-23724.

-25 Lee-Kwon W, Kim JH, Choi JW, Kawano K, Cha B, Dartt DA, Zoukhri D, Donowitz M: Ca ${ }^{2+}$-dependent inhibition of nhe3 requires pkc alpha which binds to e3karp to decrease surface nhe3 containing plasma membrane complexes. Am J Physiol Cell Physiol 2003;285:C1527-1536.

-26 Peterson JR, Lebensohn AM, Pelish HE, Kirschner MW: Biochemical suppression of small-molecule inhibitors: A strategy to identify inhibitor targets and signaling pathway components. Chem Biol 2006;13:443-452.

27 Donowitz M, Cha B, Zachos NC, Brett CL, Sharma A, Tse CM, Li X: Nherf family and nhe3 regulation. J Physiol 2005;567:3-11.

28 Sarker R, Valkhoff VE, Zachos NC, Lin R, Cha B, Chen TE, Guggino S, Zizak M, de Jonge H, Hogema B, Donowitz M: Nherf1 and nherf2 are necessary for multiple but usually separate aspects of basal and acute regulation of nhe3 activity. Am J Physiol Cell Physiol 2011;300:C771-782.

29 Zachos NC, Li X, Kovbasnjuk O, Hogema B, Sarker R, Lee LJ, Li M, de Jonge H, Donowitz M: Nherf3 (pdzk1) contributes to basal and calcium inhibition of nhe3 activity in caco-2bbe cells. J Biol Chem 2009;284:23708-23718.

30 Levine SA, Nath SK, Yun CH, Yip JW, Montrose M, Donowitz M, Tse CM: Separate c-terminal domains of the epithelial specific brush border $\mathrm{Na}^{+} / \mathrm{H}^{+}$exchanger isoform nhe3 are involved in stimulation and inhibition by protein kinases/growth factors. J Biol Chem 1995;270:13716-13725.

-31 Zachos NC, van Rossum DB, Li X, Caraveo G, Sarker R, Cha B, Mohan S, Desiderio S, Patterson RL, Donowitz M: Phospholipase c-gamma binds directly to the $\mathrm{Na}^{+} / \mathrm{H}^{+}$exchanger 3 and is required for calcium regulation of exchange activity. J Biol Chem 2009;284:19437-19444.

-32 Tu S, Wu WJ, Wang J, Cerione RA: Epidermal growth factor-dependent regulation of cdc42 is mediated by the src tyrosine kinase. J Biol Chem 2003;278:49293-49300.

-33 Wang LH, Rothberg KG, Anderson RG: Mis-assembly of clathrin lattices on endosomes reveals a regulatory switch for coated pit formation. J Cell Biol 1993;123:1107-1117.

-34 Larkin JM, Brown MS, Goldstein JL, Anderson RG: Depletion of intracellular potassium arrests coated pit formation and receptor-mediated endocytosis in fibroblasts. Cell 1983;33:273-285.

-35 Mayor S, Pagano RE: Pathways of clathrin-independent endocytosis. Nat Rev Mol Cell Biol 2007;8:603-612.

-36 Prosser DC, Wendland B: Conserved roles for yeast rho1 and mammalian rhoa gtpases in clathrinindependent endocytosis. Small GTPases 2012;3:229-235.

-37 Khurana S, Kreydiyyeh S, Aronzon A, Hoogerwerf WA, Rhee SG, Donowitz M, Cohen ME: Asymmetric signal transduction in polarized ileal $\mathrm{Na}^{+}$-absorbing cells: Carbachol activates brush-border but not basolateralmembrane pip2-plc and translocates plc-gamma 1 only to the brush border. Biochem J 1996;313:509-518.

-38 Riquier AD, Lee DH, McDonough AA: Renal nhe3 and napi2 partition into distinct membrane domains. Am J Physiol Cell Physiol 2009;296:C900-910.

-39 Yang LE, Maunsbach AB, Leong PK, McDonough AA: Differential traffic of proximal tubule $\mathrm{Na}^{+}$transporters during hypertension or pth: Nhe3 to base of microvilli vs. Napi2 to endosomes. Am J Physiol Renal Physiol 2004;287:F896-906.

40 Yip KP, Wagner AJ, Marsh DJ: Detection of apical $\mathrm{Na}^{+} / \mathrm{H}^{+}$exchanger activity inhibition in proximal tubules induced by acute hypertension. Am J Physiol Regul Integr Comp Physiol 2000;279:R1412-1418.

41 Zou Z, Chung B, Nguyen T, Mentone S, Thomson B, Biemesderfer D: Linking receptor-mediated endocytosis and cell signaling: Evidence for regulated intramembrane proteolysis of megalin in proximal tubule. J Biol Chem 2004;279:34302-34310.

42 Scarselli M, Donaldson JG: Constitutive internalization of g protein-coupled receptors and g proteins via clathrin-independent endocytosis. J Biol Chem 2009;284:3577-3585.

43 Grant BD, Donaldson JG: Pathways and mechanisms of endocytic recycling. Nat Rev Mol Cell Biol 2009;10:597-608.

44 Eyster CA, Cole NB, Petersen S, Viswanathan K, Fruh K, Donaldson JG: March ubiquitin ligases alter the itinerary of clathrin-independent cargo from recycling to degradation. Mol Biol Cell 2011;22:3218-3230. 